\title{
Superior Vena Cava Doppler Flow Changes in Superior Vena Cava Syndrome
}

\author{
Yun You Duan and Li Jun Yuan \\ Fourth Military Medical University \\ China
}

\section{Introduction}

Two major topics were covered in this chapter. The first was to explore a method of establishing a rabbit model of SVCS by injecting VX2 tumor cell suspension transcutaneously under ultrasound guidance, and to observe the radiotherapeutic effects by SVC Doppler flow changes. The establishment of this model would offer an experimental evidence for better diagnosis, treatment and follow-up observation of SVCS. The second was to investigate the evolution of the SVC Doppler flow changes in patients with SVCS and its value in assessing clinical therapeutic effects in these patients.

An animal SVC obstruction model would be of use in further studies directed at optimizing the therapeutic strategies for original tumors causing SVC syndrome in patients, yet no detailed information about the establishment of an animal SVC obstruction (SVCO) model can be found in the literature. It has been reported that VX2 tumor cell could be successfully inoculated into multiple organs to induce malignant tumors in rabbits (De Crespigny et al, 1999; Goldberg et al, 1999; Ishida et al, 2000), and various imaging modalities has been adopted to assess the effect of this inoculation (Kim et al, 2000; Liu et al, 2001). We hypothesize that a rabbit model with SVCO could be established by inoculating VX2 tumors cells into the areas around the SVC.

Superior vena cava syndrome (SVCS) is a clinical expression of obstruction of blood flow through the SVC, and more than $80 \%$ cases are caused by malignant tumors. Though contrast-enhanced spiral or multi-slice CT is now able to identify accurately the site of occlusion or stenosis, it has been greatly limited by its high cost and radiation and thus is not appropriate for follow-up observations. In contrast, ultrasound is safe, reproducible, and relatively inexpensive. Doppler ultrasonography has been successfully used to assess the rabbit SVC obstruction model. We hypothesize that Doppler flow patterns of SVC could be applied for assessing the severity of SVCS and its therapeutic effects.

\section{Objective}

There are three objectives for the animal study. The first is to study the feasibility of establishing a model of SVCO in rabbits by infusing VX2 tumor cell suspension transcutaneously with ultrasound guidance, and to evaluate the applications of this animal model. The second is to study morphologic and hemodynamic changes of superior vena cava (SVC) in rabbits with SVCO using two-dimensional and Doppler ultrasound and to 
explore the relationship between the tumor size in specimen and the two-dimensional and Doppler ultrasonographic characteristics. The third is to analyze the ultrasonographic characteristics and histopathological changes after radiotherapy in rabbits with SVCO, and to provide useful information for assessing the SVC syndrome therapeutic effect in clinic.

The objective of the human study was to evaluate the Doppler SVC's flow patterns and their value in assessing SVCS.

\section{Materials and methods}

\subsection{Animals model establishment}

Fifteen adult healthy New Zealand White rabbits were enrolled in this study. Anesthesia was performed by injecting Ketamine Hydrochloride into the posterior leg muscles of rabbits at a dose of $30 \mathrm{mg} / \mathrm{kg}$. The VX2 tumor cell suspension was prepared under the sterile condition. About $0.1 \mathrm{ml}$ tumor cell suspension was infused transcutaneously in front of SVC and close to its anterior wall guided by ultrasound. The SVC morphology and hemodynamics as well as the tissues around SVC were examined with two-dimensional and Doppler ultrasonography once every 3 days from the 9th day after the injection of tumor cell suspension till the natural death of the rabbits. These findings were compared with those by CT and digital subtraction angiography (DSA).

\subsection{Ultrasonographic examinations}

One rabbit did not develop tumor after the injection of VX2 tumor cell suspension and thus excluded. The rest of the fourteen rabbit models of SVCO were examined by twodimensional and Doppler ultrasonography using Sequoia 512 computed ultrasonograph with the probe of $7 \mathrm{v} 3 \mathrm{c}$.

The sizes of the tumor and the SVC morphology and hemodynamics were observed with the transducer placed in the right supraclavicular region view using Sequoia 512 computed ultrasonograph once every 3 days from the 12th day after the injection, and compared with the ultrasonographic findings before injection. The ultrasonographic findings were also compared with those of CT and autopsy findings, respectively.

\subsection{Radiotherapy for rabbits with SVCO due to VX2 tumor injection}

Thirteen survived rabbits with SVCO due to VX2 tumor were enrolled in this study. The tumors in mediastina were exposed one minute with 2gy everyday by SIEMNS MEVATRON 6745, which was 10 times in all. Before the radiotherapy and On the 10, 17 and 24 day after radiotherapy, the sizes of the tumor and SVC, SVC velocities and echo characteristics in the 13 rabbits were detected with the transducer placed in the right supraclavicular region using Sequoia 512 computed ultrasonograph. The mediastina neoplasm tissue before and after radiotherapy were sampled for HE staining and TUNEL for analysis of the number of the apoptotic cells.

\subsection{Patients}

Forty-two patients ( 26 females and 16 males) with SVCS caused by neoplasm (34 with right upper lung cancer and 8 with mediastinal tumor), aged from 22 to 56 years old were included in this study when they were scheduled for radiotherapy and/ or chemotherapy treatment from January 2000 to June 2005. All the patients had upper extremity and facial swelling at initial diagnosis. Twenty volunteers ( 15 males and 5 females) aged 23-52 years 
with no history of cardiac and pulmonary diseases were recruited as controls. All informed consents of the patients were acquired.

\subsection{Methods of human study}

Acuson Sequoia 512 ultrasonograph equipped with7V3C transducer was used. Electrocardiogram and respiratory curve were recorded simultaneously. The following procedures were in accordance with the ethical standards of the committee on human experimentation of the institution and approved by Tangdu Hospital committee.

Right supraclavicular approach. Patients took a supine position. With the transducer placed in the fossa between the sterna and clavicular heads of the sternomastoid muscle, the upper part of the SVC and its adjacent structures were fully displayed.

Subcostal approach. Patients took a supine position. With the transducer placed in the subcostal region, the lower part of SVC was displayed.

The SVC spectra were recorded. The flow velocities of the two forward waves, systolic wave (S) and diastolic wave (D) and the two reversed waves during ventricular and atrial contraction (VR and AR), were measured. The variation of these flow velocities with cardiac cycle and respiration was analyzed with SPSS software. All the patients were followed up for more than 11 months.

\section{Results}

\subsection{The success rate of establishing SVCO rabbit models}

Fourteen rabbits were found to have the tumors para-SVC and/ or in the SVC cavity by ultrasonography. One rabbit dropped off because no tumor grew until 42nd day after the infusion. The success rate of developing rabbit SVCO model was about 93.33\%.

\subsection{Two-dimensional ultrasonographic findings of SVCO}

The diameters of the tumors were $(80.70 \pm 4.28) \mathrm{mm}$. With the tumor growing, the lumen of SVC was deformed and narrowed, and the wall of SVC was disrupted shown by twodimensional ultrasonography (Figure 1 and 2). The tumor size growth was linearly correlated with time, and the correlation coefficient was 0.9855 (Figure 3). The tumor diameter by ultrasound was similar to the diameter by autopsy $((80.70 \pm 4.28 \mathrm{~mm}$ vs. $82.16 \pm 3.41 \mathrm{~mm}, \mathrm{t}=0.998, P=0.327405)$.

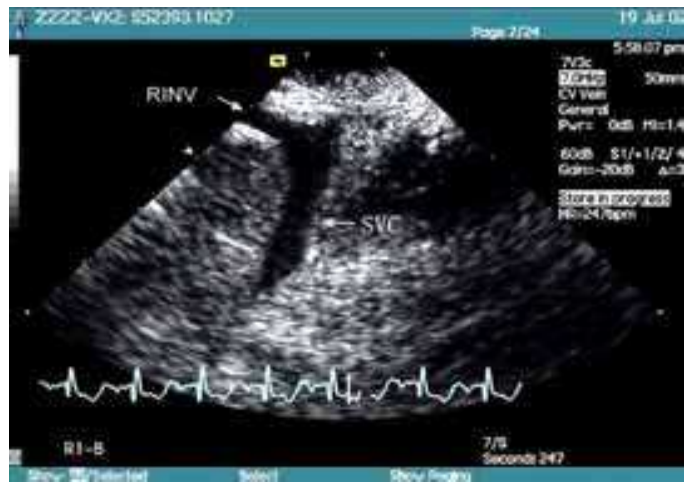

Fig. 1. Two-dimensional ultrasonography of normal SVC 


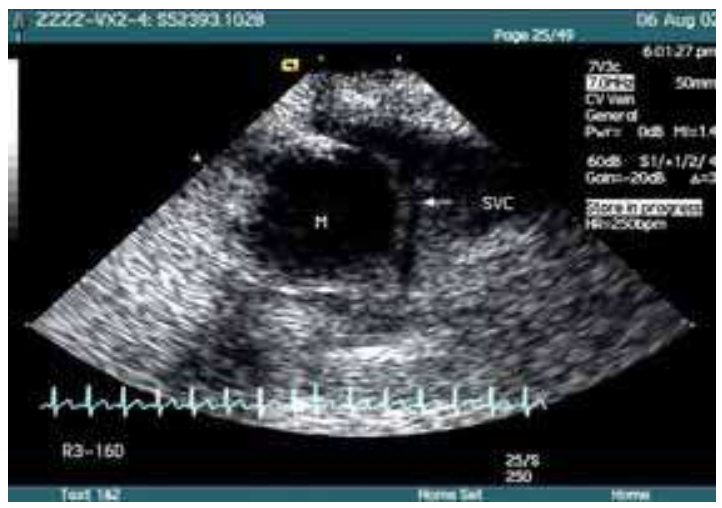

Fig. 2. Two-dimensional ultrasonography of a rabbit with SVCO

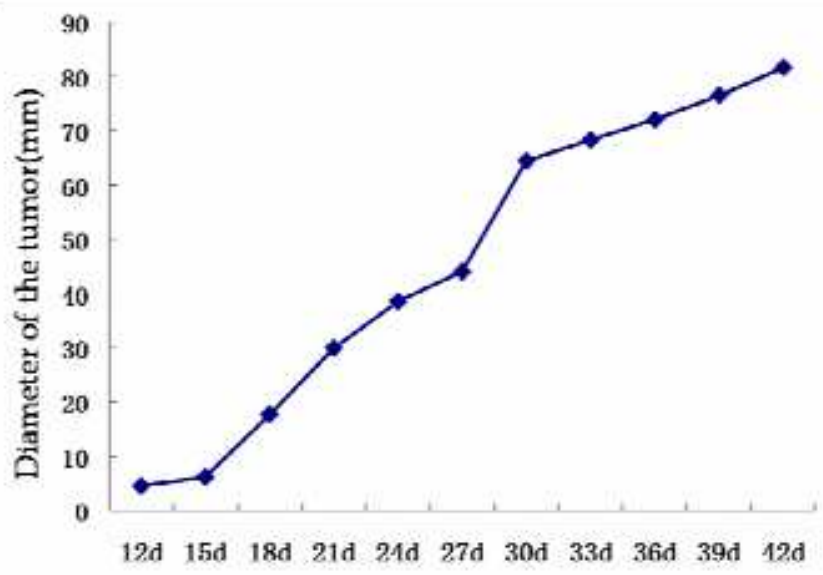

Fig. 3. Tumor growing with time

\subsection{SVCO development}

All the 14 rabbits developed SVCO. In eight rabbits, SVC was found to be oppressed by twodimensional ultrasonography on the $15^{\text {th }}$ day after infusion, Aliasing mosaic flow signals and high flow velocity spectra in SVC were demonstrated by Doppler ultrasonography. In another 4 rabbits, the stenotic blood flow velocities were displayed on the $18^{\text {th }}$ day after infusion. In the last 2 rabbits, the abnormal blood flow was seen on the 21st and the 24th day, respectively.

\subsection{The different stages of SVCO}

The development of SVCO could be divided into three stages. Early stage: About 1 week after. The tumor area was $0.5 \mathrm{~cm}^{2} \sim 3 \mathrm{~cm}^{2}$. The echotexture was hpyoechoic and evenly distributed, the shape of the tumor was regular with pseudocapsule. SVC was compressed, but the SVC wall was relatively intact (Figure 4). Mid stage: the tumor area was $3.1 \mathrm{~cm}^{2} \sim 6.0$ 
$\mathrm{cm}^{2}$. Most of the tumors still were hypoechoic echotexture. Hyperechoic textures could be seen within some tumors. T shape of the tumor was not regular. At this stage, the SVC was oppressed and its course became abnormal; the lumen of SVC was narrowed, and the wall was infiltrated by tumors (Figure 5). Late stage: The tumor area reached more than $6.1 \mathrm{~cm}^{2}$, and the shape was dramatically irregular. Mixing echotexture was seen, but was mainly hypoechoic. SVC was severely oppressed and correlated well with the size of the tumor (Figure 6). The ultrasonographic findings of SVCO at the late stage correlated well with those findings by CT and the autopsy.

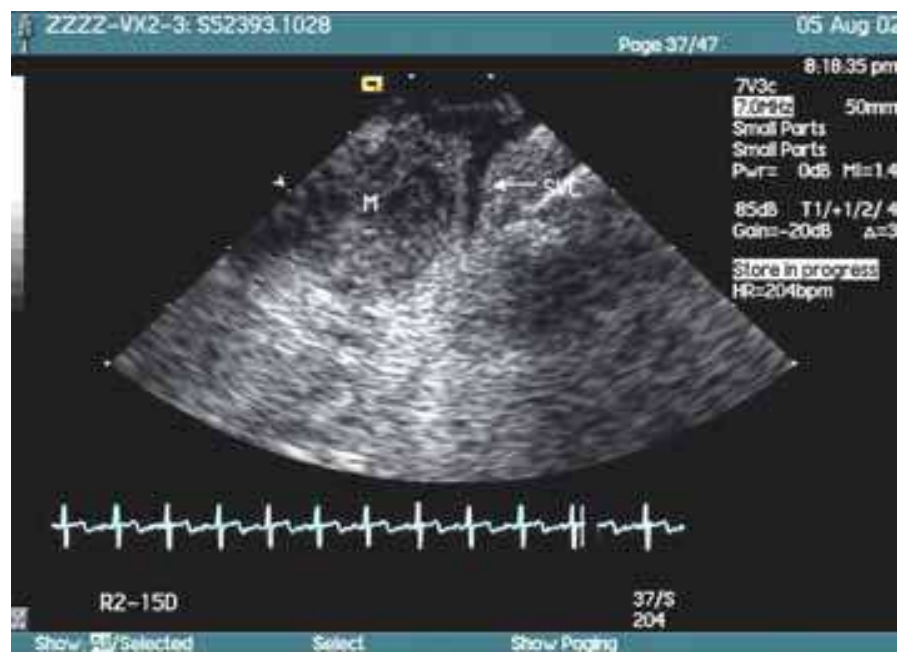

Fig. 4. SVC being compressed by a mass (M) in a rabbit with SVCO. The wall of SVC is intact

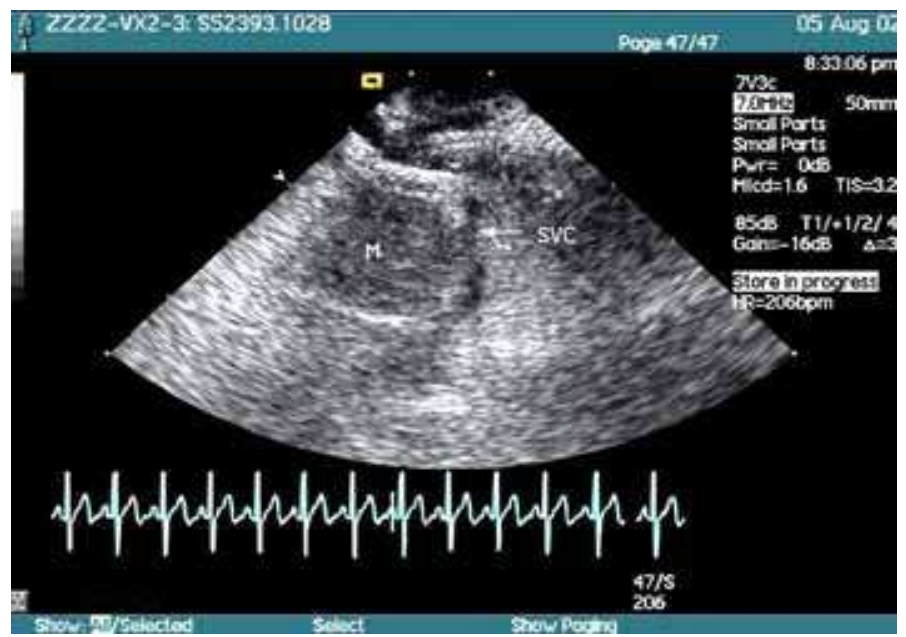

Fig. 5. SVC being compressed in a rabbit with SVCO. The wall of SVC is infiltrated by the mass $(\mathrm{M})$ 


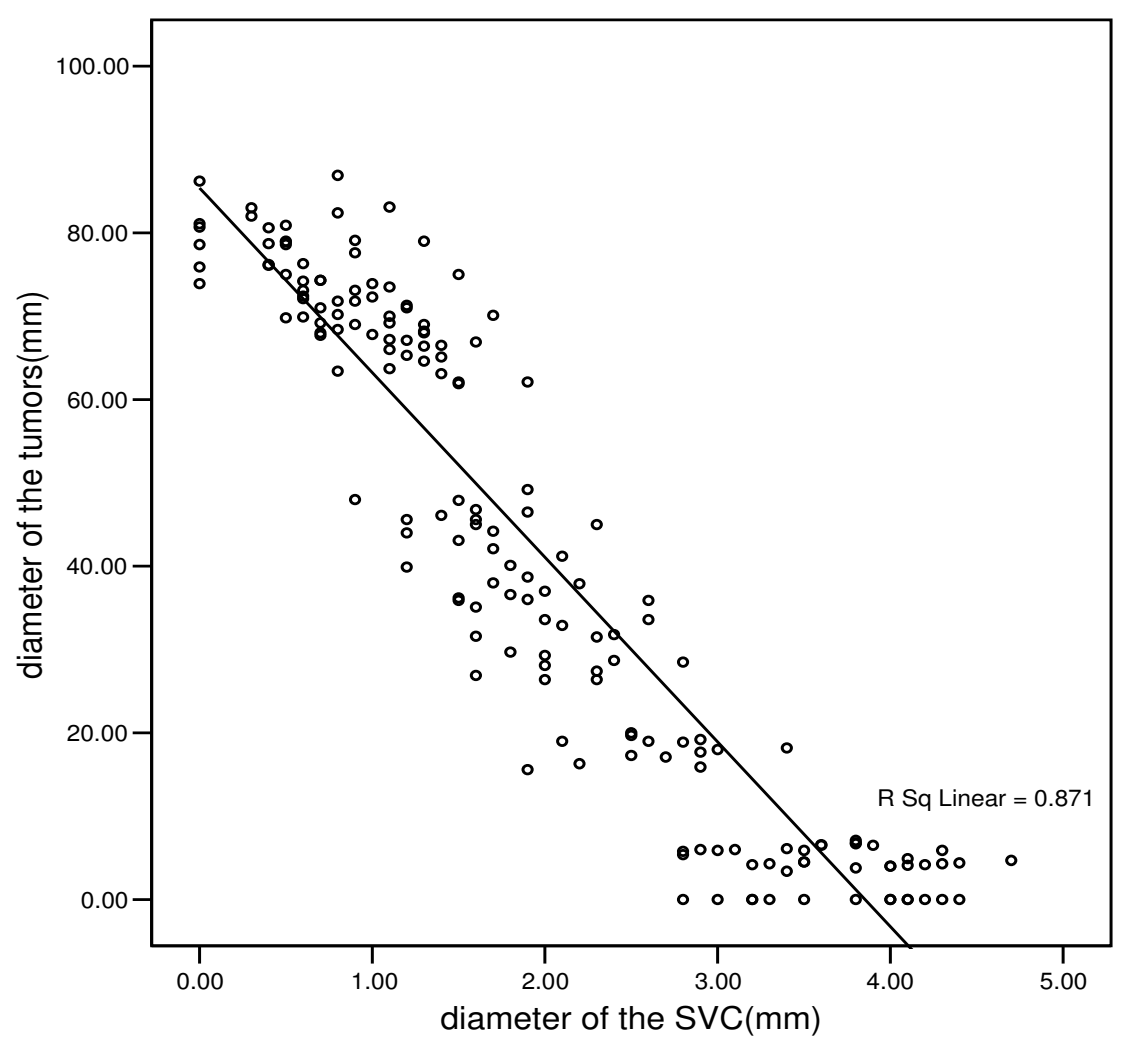

Fig. 6. Relationship between the tumor size and the SVC diameter

\subsection{Doppler ultrasonographic findings of SVCO}

In SVCO, the color Doppler ultrasound showed mosaic or weak or even no flow signals within SVC (Figure 7). In normal rabbits, the pulsed Doppler ultrasound showed laminar flow in SVC (Figure 8); while in rabbits with SVCO, the SVC flow was turbulent and the spectral window was disappeared (Figure 9). The peak flow velocities of SVC waves were less influenced by respiratory cycle in SVCO compared to normal (Figure 10). SVC flow velocities significantly increased during early and mid stages of SVCO (Table 1).

\begin{tabular}{lllll}
\hline & $\mathrm{S}$ & $\mathrm{D}$ & $\mathrm{VR}$ & $\mathrm{AR}$ \\
\hline Early and middle stages & $78.25 \pm 14.97$ & $59.68 \pm 13.16$ & $19.22 \pm 4.99$ & $17.44 \pm 2.67$ \\
Late stage & $33.71 \pm 18.90$ & $33.55 \pm 20.03$ & $10.53 \pm 3.27$ & $9.33 \pm 1.58$ \\
$\mathrm{~T}$ & 6.912 & 4.079 & 5.450 & 9.781 \\
$\mathrm{P}$ & $<0.0001$ & 0.0004 & $<0.0001$ & $<0.0001$
\end{tabular}

Table 1. SVC flow velocities changes at different stages of SVCO $(\bar{x} \pm s, \mathrm{~cm} / \mathrm{s})$ 


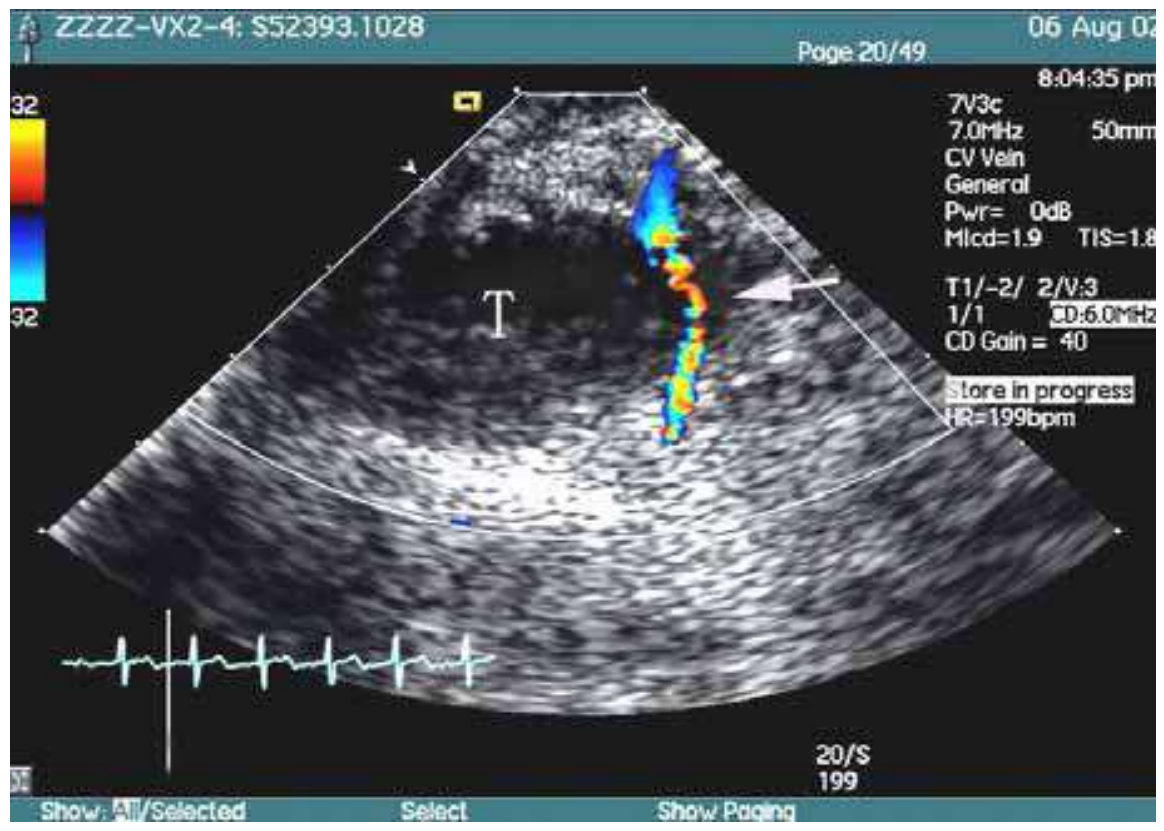

Fig. 7. Mosaic and thread-like color Doppler flow signals were seen in SVC in a rabbit with SVCO by color Doppler ultrasonography. T: tumor

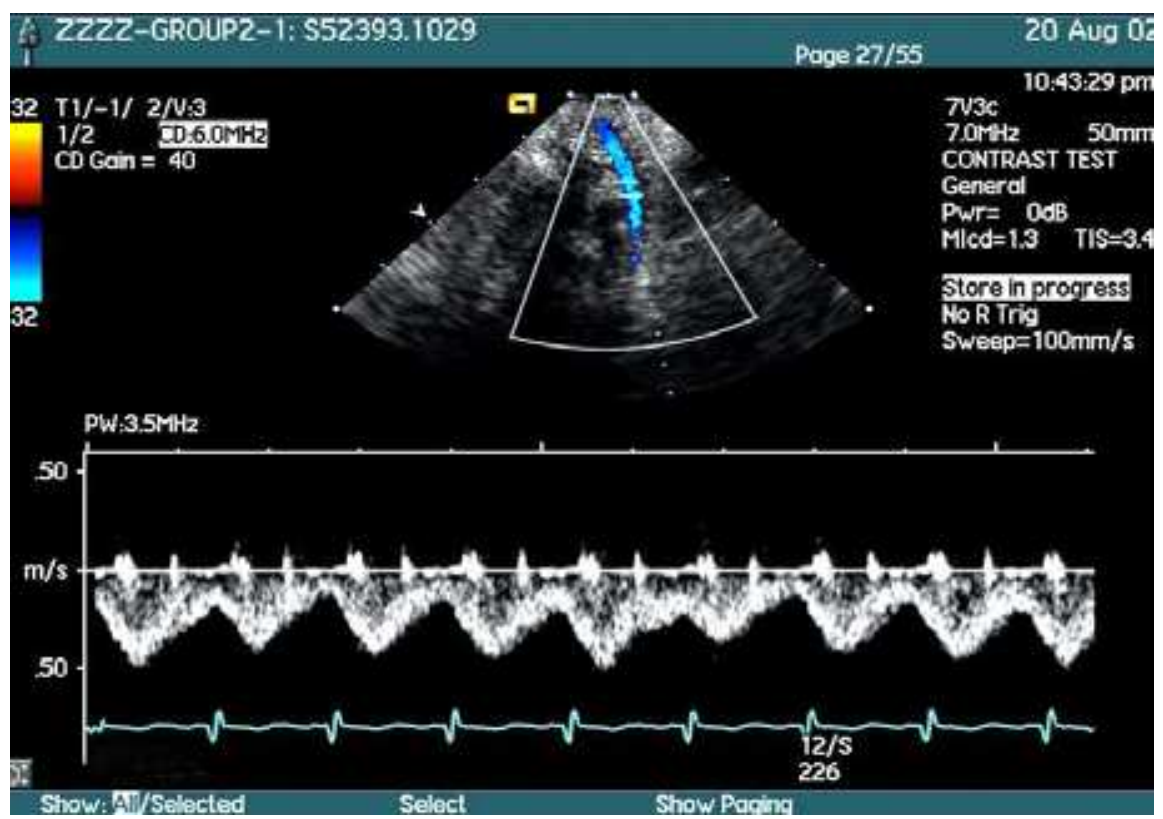

Fig. 8. Normal SVC Doppler flow 


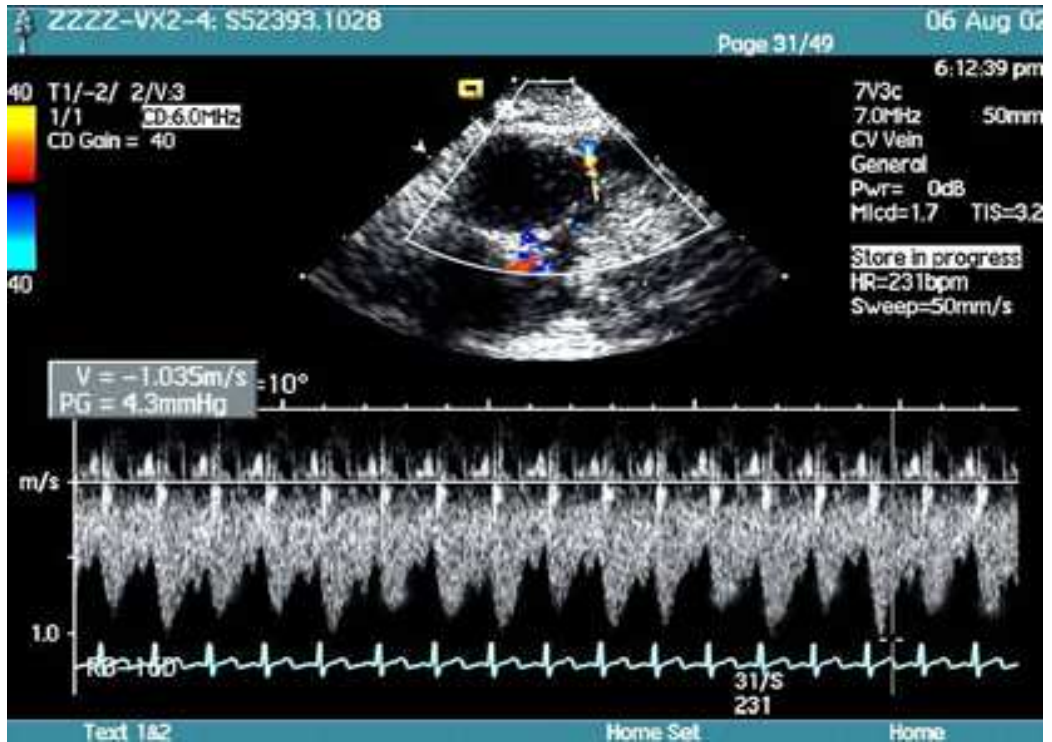

Fig. 9. Increased SVC Flow velocities in the same rabbit as shown in figure 7

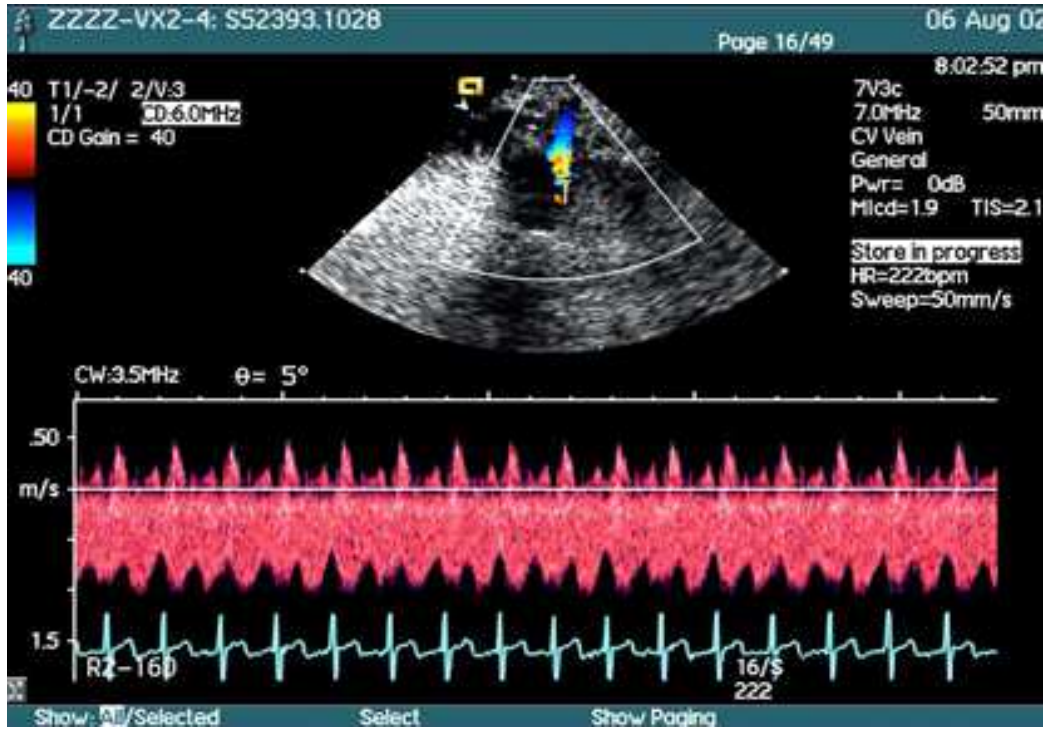

Fig. 10. Peak velocities of SVC waves were less influenced by respiratory cycle than normal

\subsection{Evaluation of radiotherapeutic effect in SVCO rabbits}

The tumor showed a tendency to get smaller after radiotherapy. The echotexture of the tumor partly turned to be hyperechoic. The diameter of SVC become bigger and the flow velocities decreased at the site of tumor compared with that before the radiotherapy (Figure 
11 and 12). The tumor diameter, SVC diameter and velocity changes after radiotherapy were shown in table 2.

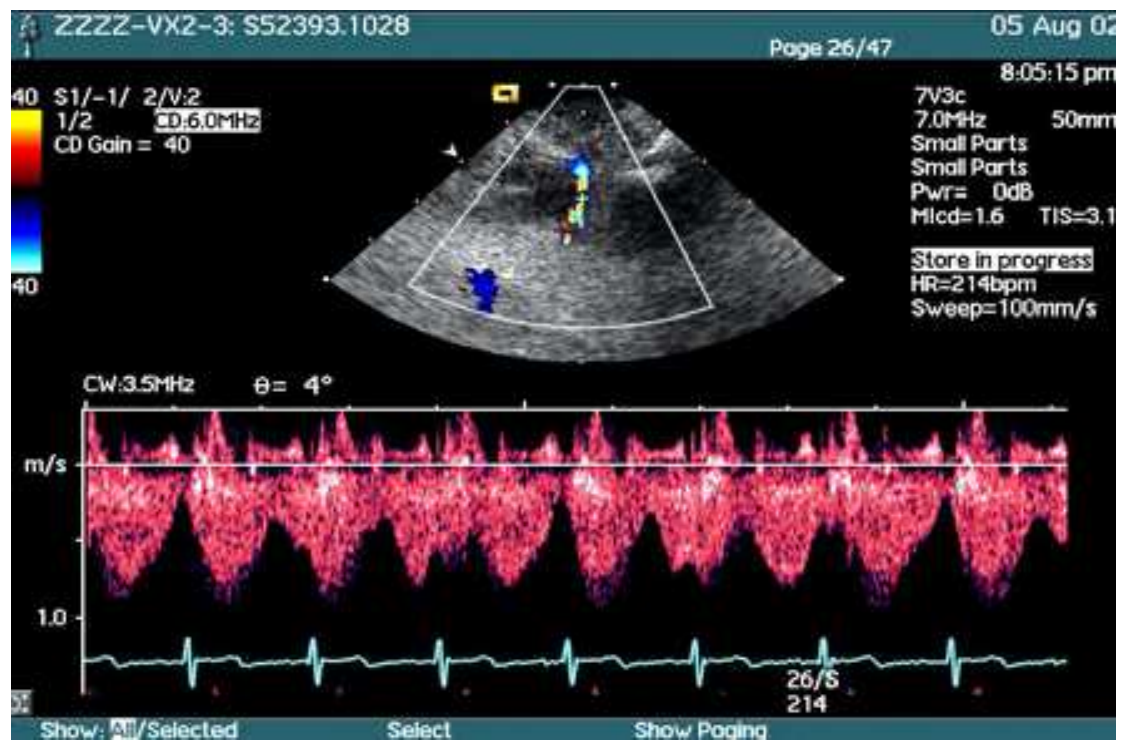

Fig. 11. SVC Doppler flow waveforms before the radiotherapy

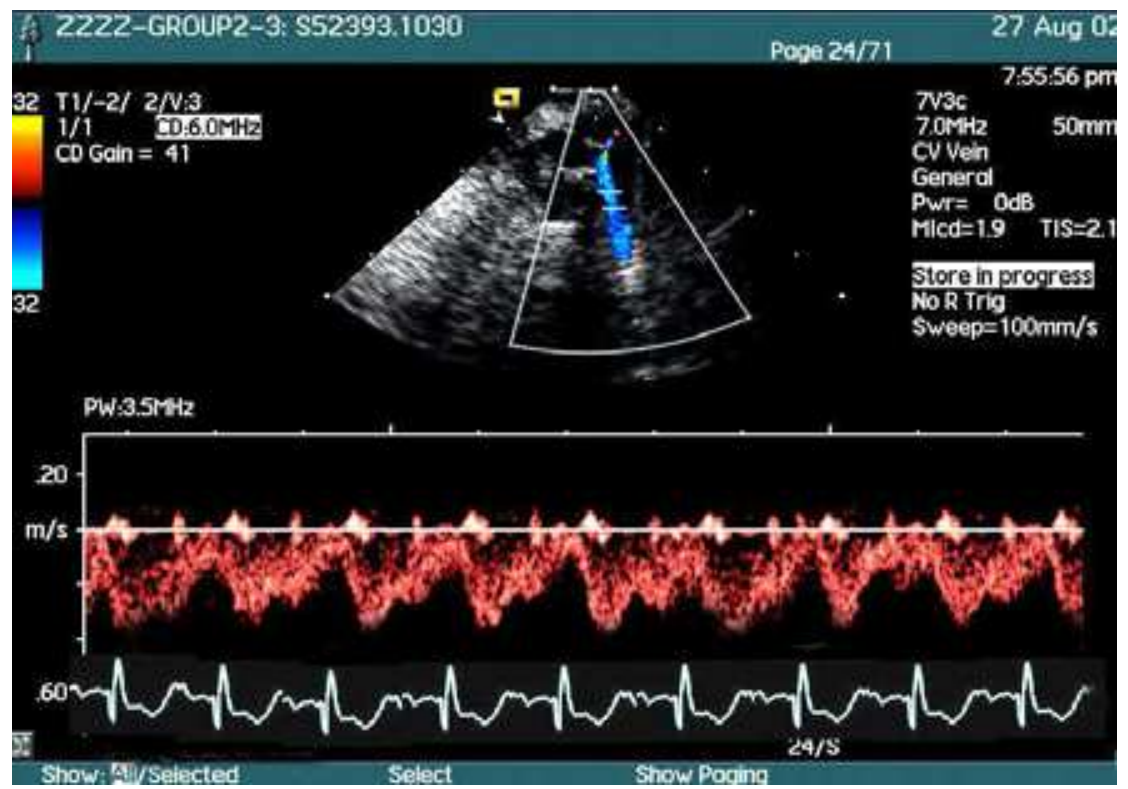

Fig. 12. SVC Doppler flow velocities decreased after radiotherapy from the same patient shown in figure 11 

in Doppler Echocardiography, Computed Tomography and Nuclear Cardiology

\begin{tabular}{llll}
\hline & $\mathrm{D} 1(\mathrm{~mm})$ & $\mathrm{D} 2(\mathrm{~mm})$ & SysVmax $(\mathrm{cm} / \mathrm{s})$ \\
\hline Before radiotherapy & $17.86 \pm 1.12$ & $2.65 \pm 0.32$ & $87.38 \pm 12.94$ \\
10d after radiotherapy & $17.64 \pm 1.08$ & $2.78 \pm 0.37$ & $84.65 \pm 11.46$ \\
17d after radiotherapy & $17.13 \pm 1.18$ & $2.94 \pm 0.93$ & $81.37 \pm 11.50$ \\
24d after radiotherapy & $16.38 \pm 1.60$ & $3.23 \pm 0.28$ & $77.55 \pm 12.34$ \\
\hline
\end{tabular}

Table 2. Tumor diameter (D1), SVC diameter (D2) and systolic maximal flow velocity (SysVmax) changes after radiotherapy

HE staining and TUNEL assay showed that the number of apoptotic cells in the tumor was much more than that before the radiotherapy (Figure 13\& 14) $(P<0.01)$.

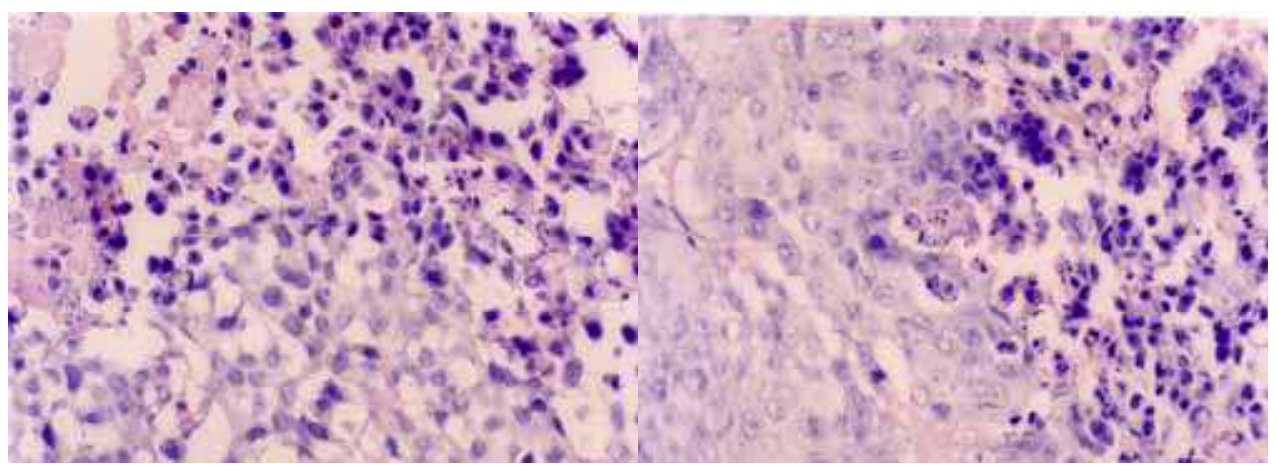

Fig. 13. HE staining showing that the apoptotic cells increased after radiotherapy (Right) compared to those before (Left)

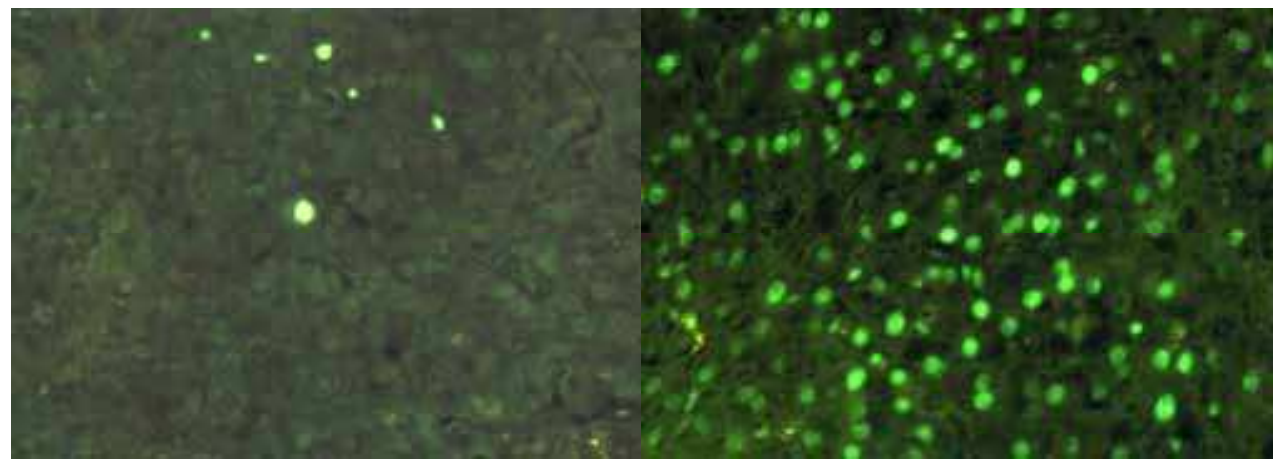

Fig. 14. TUNEL assay showing that the apoptotic cells increased after radiotherapy (Right) compared to those before (Left)

\subsection{SVC Doppler flow in patients with SVCS}

Different from healthy subjects, where laminar flow was demonstrated (Figure 15), the SVC flow spectra in patients with mild SVCS showed turbulent flow (Figure 16) and the spectral window was disappeared. In patients with moderate degree of SVCS, the distinct biphasic forward waves (S- and D-waves) of SVC were lost (Figure 17). In addition, we found that 
the smaller of the VR- and AR-waves were, the farther the oppressed segment of SVC was aw ay from the right atrium.

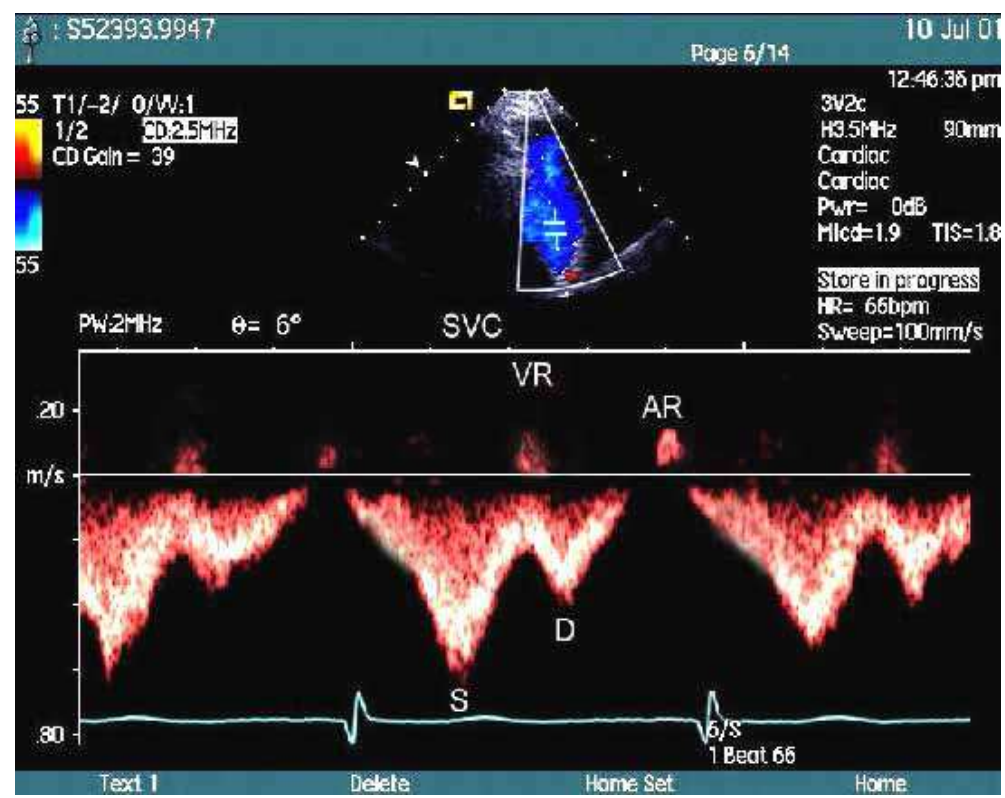

Fig. 15. SVC Doppler flow spectra in healthy subjects. Doppler interrogation of the SVC shows systolic and diastolic phases of flow (S- and D-waves) tow ard the heart and late ventricular systolic and atrial systolic phases of backward flow (VR- and AR-waves)

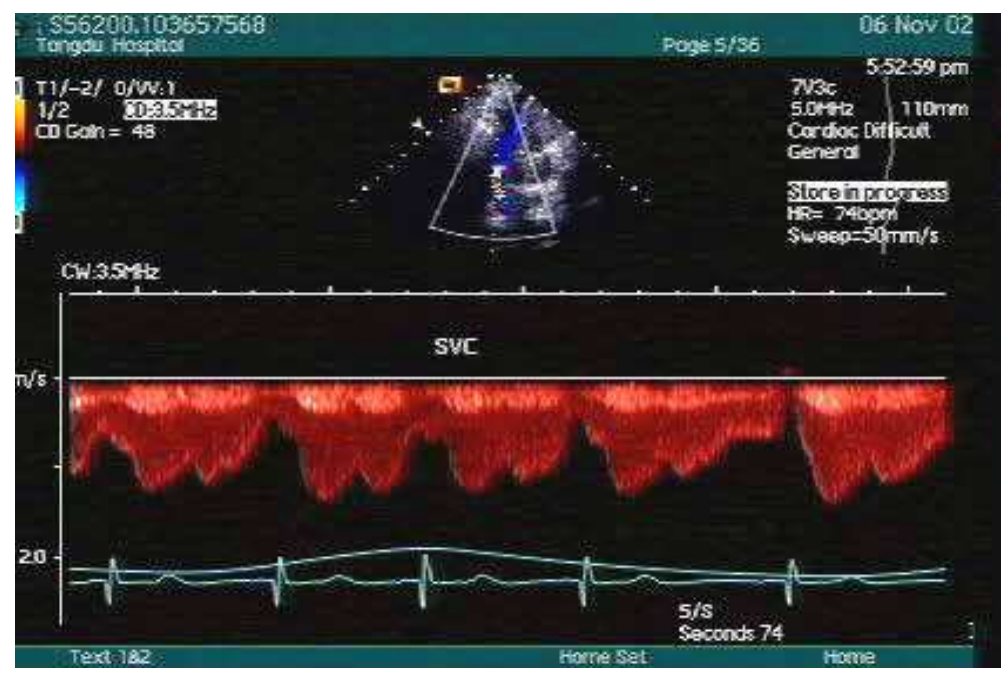

Fig. 16. SVC Doppler flow spectra in mild SVCS. The forward waves showed high velocity and no distinct spectral window 


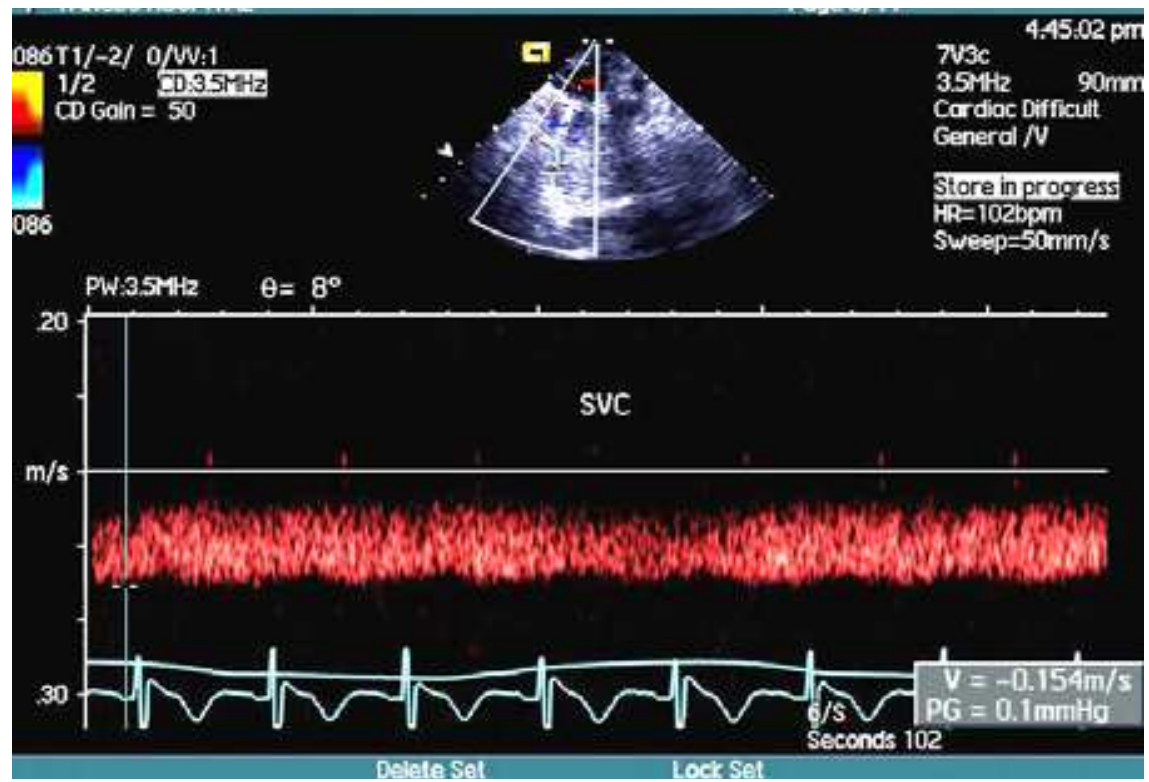

Fig. 17. SVC Doppler flow spectra in severe SVCS. The S, D AR, and VR waves could not be identified

The RVI in SVCS group were much lower than those in the control group (S-wave: $1.9 \pm 3.6 \%$ vs. $16.34 \pm 8.96 \%, \mathrm{P}=0.0003$; $\mathrm{D}$-wave: $2.80 \pm 1.23 \%$ vs. $26.32 \pm 42 \%, \mathrm{P}=0.0087$ ). After treatment, the flow velocities of SVC decreased significantly with each month and the RVI was significantly increased compared to those before the treatment.

\section{Comments}

The animal study demonstrated that SVCO rabbit model could be easily established transcutaneously with the guidance of ultrasound. With VX2 tumor growing, SVC was oppressed and/ or infiltrated by the tumor tissues and ultimately resulted in the blockage of the SVC, and developed SVCO. The pathogenesis of SVCO in rabbits was similar to that of SVCO in humans, indicating that this rabbit model of SVCO caused by VX2 tumor could be an ideal model of SVCO.

Two-dimensional ultrasonography could be used to demonstrate the morphologic changes of the tumor and SVC. Color Doppler could sensitively detect the abnormal blood flow of SVC in rabbits with SVCO. It could be mosaic, weak or even no flow signals depending on the different stages and severity of SVCO. Doppler flow spectra of SVC showed less respiratory influences on the flow velocities in rabbits with SVCO.

Linear accelerator radiotherapy could lead to cell apoptosis of the VX2 tumor transplanted besides SVC. HE staining and TUNEL assay showed increased number of apoptotic cells of the tumor tissue compared to that before radiotherapy. With the apoptosis of the tumor cells, the echotexture of the tumor partly turned to be hyperechoic and the tumor itself was getting smaller. Thereby the oppression severity of the SVC was decreased, the diameter of SVC was getting bigger, and finally the obstruction was getting resolved. 
With the release of the obstruction after radiotherapy, the respiratory variation of the SVC Doppler flow velocities could be recovered and the peak flow velocities of SVC decreased gradually after the treatment shown by Doppler ultrasonography. However, the tumor size, SVC diameter and the flow velocity could not completely get back to normal after treatment. This suggests that Linear accelerator radiotherapy could not inactivate VX2 tumor completely, which might be related to the high grade of malignancy, poor proliferation of the VX2 tumors.

The human study demonstrates that respiratory variations of SVC Doppler flow velocities are significantly decreased in patients with SVCS, but could be recovered gradually after treatment, indicating that respiratory variations of Doppler flow changes of SVC correlate well with the severity of SVCS, and may be used to assess the therapeutic effects of SVCS. The SVC Doppler flow patterns in patients with SVCS were characterized with fill-in and broaden spectra, nondistinct outlines. The D-wave was unable to return to the baseline at end diastole, which was consistent with previous studies (Yano\&Shimada, 1997). When the patients' condition improved after treatment, , the pressure gradient at the stenotic segment of SVC also decreased, indicating that SVC hemodynamic changes was in accordance with the clinical courses of SVCS (Behar et al., 2001; Tighe et al., 2000).

It has been well known that, respiration had significant influence on SVC flow velocities in healthy subjects. The S- and D-wave peak velocities are much greater on inspiration compared with those on expiration because the decreased intrathoracic pressures during inspiration causes increased venous return (Jellinek et al., 2000; Vieillard et al., 2001). In patients with SVCS, However, the obstruction prevents the conduction of the intrathoracic pressure changes into the right atrium and to SVC, resulting in decreased or even diminished respiratory variations of S- and D-wave flow velocities. Once this obstruction is released after treatment, the SVC Doppler flow velocities would decrease and the respiratory variations of these flow velocities would be back.

\section{Conclusions}

Rabbit model of SVCO caused by VX2 tumor could be an ideal model of SVCO for clinical study. Doppler ultrasound is the first method of choice for assessing the hemodynamics of SVCO.

Normally, respiration had significant influence on SVC morphology and dynamics in healthy subjects. This influence would become less in the patients with SVCS, suggesting that SVC Doppler spectra could reflect the severity of SVCS.

\section{References}

Behar DM., Reisner SA., Ben-Ami H (2001). Superior vena cava syndrome diagnosed by transeso phageal echocardiography. Isr Med Assoc J, Vol 3, No.6, pp. 457-460.

De Crespigny AJ., How ard D., D'Arceuil H., et al (1999). Dynamic contrast-enhanced MRI of Implanted VX2 tumors in rabbit muscle: comparison of Gd-DTPA and NMS60. Magn Reson Imaging, Vol. 17, No. 9, pp. 1297-1305.

Goldberg SN., Walovitch RC., Straub JA., et al (1999). Radio-frequency-induced coagulation necrosis in rabbits: immediate detection at US with a synthetic microsphere contrast agent. Radiology, Vol. 213, No. 2, pp. 438-444. 
Ishida H., Murata N., Yamada H., et al (2000). Pneumoperitoneum with carbon dioxide enhances liver metastases of cancer cells implanted into the portal vein in rabbits. Surg Endosc, Vol 14, No. 3, pp. 239-242.

Jellinek H., Krenn H., Oczenski W, et al (2000). Influence of positive airway pressure on the pressure gradient for venous return in humans. JAppl Physiol, Vol 88, No.3, pp. 926-932.

Kim TK., Choi BI., Park SW., et al (2000). Gadolinium mesoporphyrin as an MR imaging contrast agent in the evaluation of tumors: an experimental model of VX2 carcinoma in rabbits. Am JRoentgenol, Vol 175, No. 1, pp. 227-234.

Liu JB., Goldberg BB., Merton DA., et al (2001). The role of contrast-enhanced sonography for radiofrequency ablation of liver tumors. JUltrasound Med, Vol 20, No. 5, pp. 517523.

Tighe DA., Paul שJ., Huhta $\mathbb{C}$ (2000). Superior vena caval syndrome: color flow Doppler detection of collateral venous channels. JAm Soc Echocardiogr, Vol 13, No. 8, pp. 780-784.

Vieillard Baron A., Augarde R., Prin S., et al (2001). Influence of superior vena caval zone condition on cyclic changes in right ventricular outflow during respiratory support. Anesthesiology, Vol 95, No. 5, pp. 1083-1088.

Yano S., Shimada K (1997). Changes in superior vena cava pulsed Doppler flow patterns: Possible indicator of improvement of superior vena cava syndrome due to lung cancer. Ultrasound Med, Vol 16, No. 10, pp. 707-710. 


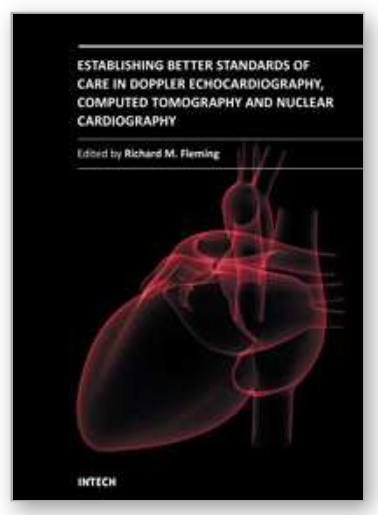

\author{
Establishing Better Standards of Care in Doppler \\ Echocardiography, Computed Tomography and Nuclear \\ Cardiology \\ Edited by Dr. Richard M. Fleming
}

ISBN 978-953-307-366-8

Hard cover, 260 pages

Publisher InTech

Published online 13, July, 2011

Published in print edition July, 2011

Since the introduction of Doppler Echocardiography, Nuclear Cardiology and Coronary CT imaging, clinicians and researchers have been searching for ways to improve their use of these important tools in both the diagnosis and treatment of heart disease. To keep up with cutting edge improvements in these fields, experts from around the world have come together in this book to provide the reader with the most up to date information to explain how, why and when these different non-invasive imaging tools should be used. This book will not only serve its reader well today but well into the future.

\title{
How to reference
}

In order to correctly reference this scholarly work, feel free to copy and paste the following:

Yun You Duan and Li Jun Yuan (2011). Superior Vena Cava Doppler Flow Changes in Superior Vena Cava Syndrome, Establishing Better Standards of Care in Doppler Echocardiography, Computed Tomography and Nuclear Cardiology, Dr. Richard M. Fleming (Ed.), ISBN: 978-953-307-366-8, InTech, Available from: http://www.intechopen.com/books/establishing-better-standards-of-care-in-doppler-echocardiographycomputed-tomography-and-nuclear-cardiology/superior-vena-cava-doppler-flow-changes-in-superior-venacava-syndrome

\section{INTECH}

open science | open minds

\section{InTech Europe}

University Campus STeP Ri

Slavka Krautzeka 83/A

51000 Rijeka, Croatia

Phone: +385 (51) 770447

Fax: +385 (51) 686166

www.intechopen.com

\section{InTech China}

Unit 405, Office Block, Hotel Equatorial Shanghai

No.65, Yan An Road (West), Shanghai, 200040, China

中国上海市延安西路65号上海国际贵都大饭店办公楼 405 单元

Phone: +86-21-62489820

Fax: +86-21-62489821 
(C) 2011 The Author(s). Licensee IntechOpen. This chapter is distributed under the terms of the Creative Commons Attribution-NonCommercialShareAlike-3.0 License, which permits use, distribution and reproduction for non-commercial purposes, provided the original is properly cited and derivative works building on this content are distributed under the same license. 